\title{
One loop matching coefficients for a variant overlap action-and some of its simpler relatives
}

\author{
Thomas DeGrand \\ Department of Physics, University of Colorado, Boulder, CO 80309 USA
}

(November 21, 2018)

\begin{abstract}
I present one-loop perturbative calculations of matching coefficients between matrix elements in continuum regulated QCD and lattice QCD with overlap fermions, with emphasis a recentlyproposed variant discretization of the overlap. These fermions have extended ("fat link") gauge connections. The scale for evaluation of the running coupling constant (in the context of the LepageMackenzie fixing scheme) is also given. A variety of results (for additive mass renormalization, local currents, and some non-penguin four-fermion operators) for naive, Wilson, clover, and overlap actions are shown.
\end{abstract}

\section{INTRODUCTION}

This paper presents one-loop perturbative calculations of matching coefficients between matrix elements computed using a spatial lattice regulator (and measured in lattice simulations) and their equivalent continuum-regulated values. The principal lattice fermion action studied is a recent formulation of overlap fermions [1]) built from a "kernel action" with nearest and next-nearest neighbor fermionic interactions and "fat link" gauge connections [2], specifically HYPblocked [3] links. Along the way, I present some new results for a number of simpler actions-naive, Wilson, and clover fermions with fat links, (including a few results for APE-blocked [4] links), and overlap actions with Wilson or clover action kernels. I also present results for the scale at which the running coupling constant is evaluated (the so-called $q^{*}$ value), using the scheme of Lepage and Mackenzie [5].

Fat link actions were originally developed [6] in order to improve the chiral properties of lattice fermions. In the context of perturbation theory, "chiral improvement" for nonchiral actions means having small additive mass renormalization, vector and axial current renormalization factors nearly equal, scalar and pseudoscalar factors also nearly equal, and suppressed mixing of four-fermion operators between different chiral sectors. Fat link actions do all these things. I have found that using these actions as kernels for overlap actions seems to have computational advantages over using thin link actions. Overlap actions with fat links have matching factors which are much closer to unity than overlap actions built of thin link fermion action kernels.

Several authors have published calculations of lattice-to-continuum matching factors for a variety of processes for the overlap action with the thin link Wilson action as its kernel (for a partial list, see [7-11]). Perturbative calculations for more complicated actions is in principle not much more difficult to do, once the Feynman rules are constructed. One just has to organize the calculation, and not carry it too far. Intermediate results for matching coefficients (particularly Table II) may be a more important part of the paper than the tables of results. These formulas are certainly not new, but in most published papers they appear embedded in action-specific discussions, and they are often hard to find until after one has rederived them.

Whether or not perturbation theory can - or should be - used to convert lattice numbers to continuum ones is a matter of debate. One sometimes sees blanket statements about the use of perturbation theory in lattice calculations, as if perturbative calculations per se were unaffected by the particular choice of lattice action. But clearly different actions have different properties when the cutoff is not taken away. At nonzero cutoff, it is a practical question for any particular action, to ask how well perturbative calculations perform. At sufficiently small renormalized coupling the matching from one scheme to another can be done with small uncertainty, the uncertainty being due to higher order terms in a perturbative expansion in the renormalized coupling.

To be specific, let's write a one loop matching coefficient for an operator which does not undergo mixing as $Z=1+\alpha_{s} /(4 \pi) A$. In today's simulations, typical values of $\alpha_{s} /(4 \pi) \simeq 0.01-0.015$. With standard actions, one often finds that the $A$ 's are large. For standard thin-link actions A is order 10-40, so the matching factor differs from unity by an amount of 0.1 to 0.6 . In the actions considered here, typically the $A$ 's are smaller than about 5 in magnitude, corresponding to a shift from unity of 0.05-0.07. One presumes, therefore, that the perturbative expansion of $Z$ is better behaved. One's results are also less sensitive to the choice of scale $q$ for $\alpha_{s}\left(q^{2}\right)$ if the coefficient is small.

A brief report of perturbation theory for Wilson and clover actions with APE-blocked links has been presented in Ref. [12]. The qualitative features of the work done here are all anticipated in that paper. Here I will focus mainly on 
HYP blocking simply because it gives more smoothing without delocalizing the action. Applications to the overlap in this work are also new. Results for staggered fermions using HYP links have recently been presented by Sharpe and Lee [13], and Lee [14] has discussed general features of perturbation theory for fat links.

In Sec. II, I describe the simple ingredients I used for lattice perturbation theory. Results of selected calculations for currents are presented in Sec. III. Appendices contain Feynman rules for the actions studied.

\section{INGREDIENTS}

\section{A. Matching and Scale Setting}

A "typical" matching coefficient between a lattice regulated quantity and a dimensionally-regulated quantity (modified minimal subtraction, $\overline{M S}$, for example), for an operator which does not undergo mixing, is $Z=1+\left(g^{2} /\left(16 \pi^{2}\right)\right) \mathcal{Z}$ where

$$
\mathcal{Z}=I \frac{F}{M S}-I_{\text {latt }}
$$

$I \frac{F}{M S}$ is the finite part of the continuum $2 \omega=4-2 \epsilon$ dimensional integral and $I^{\text {latt }}$ is a lattice integral. For a process with $N_{f}$ external fermion legs, $I_{i}=\Gamma_{i}-\Sigma_{1} N_{f} / 2$ where $\Gamma_{i}$ is the vertex renormalization and $\Sigma_{1}$ is the fermion wave function renormalization. All these quantities will be evaluated at one loop. In this work the internal momentum integration variable will always label the momentum flowing through the gluon line. I will always work at zero momentum for external particles and fermion mass $m=0$, regulating any IR (infared) divergence of the diagram with a gluon mass $\lambda$. Of course, there is nothing deep about these choices; they are made purely for expediency. $I_{\bar{M} S}$ will take the generic form

$$
I \overline{M S}=16 \pi^{2} \int \frac{d^{2 \omega} k}{(2 \pi)^{2 \omega}}\left(\mu^{2}\right)^{2 \omega} \frac{1}{k^{2}\left(k^{2}+\lambda^{2}\right)}(A+B \epsilon)=A\left\{\frac{1}{\epsilon}-\gamma_{E}+\log (4 \pi)\right\}+A \log \frac{\mu^{2}}{\lambda^{2}}+A+B .
$$

The term in curly brackets is simply discarded to give $I \frac{F}{M S}$. In the lattice integral, we can scale all dimensionful variables by appropriate powers of the lattice spacing and write

$$
I_{l a t t}=16 \pi^{2} \int_{a k} I(a k, a p, a m, a \lambda)
$$

where $\int_{a k}=\prod_{j} \int_{-\pi}^{\pi} d\left(a k_{j}\right) /(2 \pi)$ will be the symbol for integration over the (rescaled) momentum hypercube.

If $I_{\overline{M S}}$ has an $A \log \left(\mu^{2} / \lambda^{2}\right)$ term, $I_{\text {latt }}$ will have an $A \log \left(1 /\left(\lambda^{2} a^{2}\right)\right)$ IR divergence, too. It can be separated out by writing the integrand as

$$
\begin{aligned}
I_{l a t t} & =16 \pi^{2} \int_{k}\left(I(k, a p, a m, a \lambda)-A \frac{\theta\left(\pi^{2}-k^{2}\right)}{k^{2}\left(k^{2}+a^{2} \lambda^{2}\right)}\right)+16 \pi^{2} \int \frac{d^{4} k}{(2 \pi)^{4}} A \frac{\theta\left(\pi^{2}-k^{2}\right)}{k^{2}\left(k^{2}+a^{2} \lambda^{2}\right)} \\
& \equiv J+A \log \frac{\pi^{2}}{a^{2} \lambda^{2}}
\end{aligned}
$$

The first term of Eq. 4 is IR finite, and one can set $\lambda=0$ in it. Thus

$$
\mathcal{Z}=A \log \left(\mu^{2} a^{2}\right)+A\left(1-\log \pi^{2}\right)+B-J .
$$

This would be the end of the story if we did not want to choose a scale $q^{*}$ for the coupling constant in Eq. 1. This is (unfortunately) a problem whose solution involves at least a two-loop calculation (see the discussion in the Appendix of Ref. [15]). Absent such a calculation, one could either let $q^{*}$ vary over some "reasonable" range $\left(1<q^{*} a<\pi\right.$ at lattice spacing $a$, for example), or construct some physically motivated ansatz for the scale. The best known example of such a construction is that of Lepage and Mackenzie [5]: Imagine that one has some process parameterized by a

one loop integral, and assume that its higher order behavior is dominated by gluonic vacuum polarization, so

$$
\alpha \int I(q) d^{4} q \rightarrow \alpha\left(q^{*}\right) \int I(q) d^{4} q \approx \int \alpha(q) I(q) d^{4} q
$$


Expanding the coupling $\alpha(q)=\alpha\left(q^{*}\right)-\beta_{0} \alpha\left(q^{*}\right)^{2} \log \left(q^{2} / q^{* 2}\right)+\ldots$, self consistency requires that the coefficient of $\alpha\left(q^{*}\right)^{2}$ vanish, or

$$
\log \left(q^{*}\right)=\frac{\int d^{4} q \log (q) I(q)}{\int d^{4} q I(q)} \equiv \frac{\mathcal{L}_{1}}{\mathcal{Z}} \equiv\left\langle\left\langle\log \left(q^{2}\right)\right\rangle\right\rangle
$$

For the actions studied here, it often happens that the $\mathcal{Z}$ coefficient of Eq. (1) is close to zero, and the calculation of $q^{*}$ using Eq. (7) produces absurd results. In that case, I substitute the higher order expression of Hornbostel, Lepage and Morningstar [16],

$$
\log \left(q^{* 2}\right)=\left\langle\left\langle\log \left(q^{2}\right)\right\rangle\right\rangle \pm\left[-\sigma^{2}\right]^{1 / 2}
$$

with

$$
\sigma^{2}=\left\langle\left\langle\log ^{2}\left(q^{2}\right)\right\rangle\right\rangle-\left\langle\left\langle\log \left(q^{2}\right)\right\rangle\right\rangle^{2}
$$

and

$$
\left\langle\left\langle\log ^{2}\left(q^{2}\right)\right\rangle\right\rangle=\frac{\int d^{4} q \log ^{2}\left(q^{2}\right) I(q)}{\int d^{4} q I(q)} \equiv \frac{\mathcal{L}_{2}}{\mathcal{Z}}
$$

is the weighted average analogous to Eq. (7).

This is all well-defined for finite pure lattice expressions, but when the operator has an anomalous dimension, it is not obvious what to do. In that case, I evaluate Eq. 7 following a prescription learned from C. Bernard [17]. His proposal is to construct a combination of dimensionally-regulated integrals whose sum gives the term in curly brackets, and to subtract them from the integral Eq. (2) to produce a finite integral in $4-2 \epsilon$ dimensions. The $\epsilon \rightarrow 0$ limit of the subtracted integral can then be taken, leaving a UV-finite four-dimensional integral for $\Gamma \frac{F}{M S}$ which can be combined with the integrand of Eq. 4. To do this, consider the two integrals

$$
I_{1}=16 \pi^{2} \int \frac{d^{2 \omega} k}{(2 \pi)^{2 \omega}}\left(\mu^{2}\right)^{2 \omega} \frac{1}{k^{2}\left(k^{2}+\mu^{2}\right)}(A+B \epsilon)=A\left\{\frac{1}{\epsilon}-\gamma_{E}+\log (4 \pi)\right\}+A+B
$$

and

$$
I_{2}=16 \pi^{2} \int \frac{d^{2 \omega} k}{(2 \pi)^{2 \omega}}\left(\mu^{2}\right)^{2 \omega} \frac{1}{\left(k^{2}+\mu^{2}\right)^{2}}(A+B \epsilon)=A\left\{\frac{1}{\epsilon}-\gamma_{E}+\log (4 \pi)\right\}+B .
$$

If $a+b=1$ and $a(A+B)+b B=0, a I_{1}+b I_{2}$ is equal to the difference $\Gamma_{\overline{M S}}-\Gamma \frac{F}{M S}$ and we can combine the three expressions under one integral, then take the $\epsilon \rightarrow 0$ limit, to write

$$
I \frac{F}{M S}=A J_{1}+B J_{2}
$$

where, pushing the IR divergence from the $\overline{M S}$ integral into the lattice integral

$$
J_{1}=16 \pi^{2} \int \frac{d^{4} k}{(2 \pi)^{4}}\left[\frac{1-\theta\left(\pi^{2}-k^{2}\right)}{k^{2}\left(k^{2}+\lambda^{2}\right)}-\frac{1}{\left(k^{2}+\mu^{2}\right)^{2}}\right]
$$

and

$$
J_{2}=16 \pi^{2} \int \frac{d^{4} k}{(2 \pi)^{4}}\left[\frac{1}{k^{2}\left(k^{2}+\mu^{2}\right)}-\frac{1}{\left(k^{2}+\mu^{2}\right)^{2}}\right] .
$$

The integrands of $J_{1}, J_{2}$, and $J$ are then used in Eqs. 7 or 8 (with appropriate powers of $\log \left(q^{2}\right)$, of course). Naturally, the particular choice of integrals $J_{1}$ and $J_{2}$ are not unique, but because these integrals are typically small in magnitude this is not generally a practical problem. Notice that the $q^{*}$ scale depends on $\mu$. I will present results only for the case $\mu a=1$.

Presumably other prescriptions can be devised. Their results will probably only depend on the coefficients A and B in Eq. 2, so I will tabulate those parameters below for the processes considered.

The definition of $q^{*}$ for matching coefficients for operators which mix, like the electroweak penguin operators, can be made as follows:. Eq. 7 expands into a matrix equation 


$$
\log q^{* 2} \mathcal{Z}=\mathcal{L}_{1}
$$

where now $\mathcal{Z}$ and $\mathcal{L}_{1}$ are matrices. There is a basis in which $\log q^{* 2}$ is diagonal, found by solving the eigenvalue equation $\mathcal{L}_{1} \mathcal{Z}^{-1}-1 \log q^{* 2}=0$, and in the original operator basis, the matrix of scales is $\log q^{* 2}=W\left(\log q^{* 2}\right)_{\text {diag }} W^{-1}$, where $W$ is the basis-transformation matrix. Each eigenvector's scale can be translated into a coupling, and

$$
Z_{i j}=\delta_{i j}+W\left[\alpha_{s}\left(q^{*}\right) /(4 \pi)\right]_{d i a g} W^{-1} \mathcal{Z} .
$$

The higher order formula Eq. 8 does not have an obvious matrix transformation. One possibility is to rotate the higher order matrix $\mathcal{L}_{2}$ into the basis which diagonalizes $\mathcal{L}_{1} \mathcal{Z}^{-1}$, and use the diagonal entries in Eq. 8.

This is unsatisfactory, but again, the small size of $\mathcal{Z}$ and the $\mathcal{L}_{i}$ 's for fat link actions means that the spread in the $Z$-factor remains small as the coupling is varied.

\section{B. Actions and Feynman rules}

Feynman rules for ordinary (non-overlap) discretizations of the Dirac operator can be constructed using standard techniques. I will parameterize the free massive Dirac operator as

$$
d(p, m)=i \gamma_{\mu} \rho_{\mu}(p)+\lambda(p)+m ;
$$

I implicitly assume the normalization that at small $p, \rho_{\mu}(p) \simeq p_{\mu}$ and $\lambda(p)=O\left(p^{2}\right)$. (This factorization might not be appropriate for an approximate fixed-point action, designed to follow out a renormalized trajectory as the mass is varied: $\rho_{\mu}$ and $\lambda$ would both be functions of the bare mass [19].) Of course, the propagator is the inverse of $d$.

While I will not specify an explicit form for the gluon propagator in any expression, I will only present results for the Wilson gauge action. Its propagator includes an IR regulator mass $\lambda$ and gauge parameter $\xi$

$$
G_{\mu \nu}=\frac{\delta_{\mu \nu}+(\xi-1) \hat{k}_{\mu} \hat{k}_{\nu} / \hat{k}^{2}}{\hat{k}^{2}+\lambda^{2}}
$$

with $\hat{k}_{\mu}=2 / a \sin \left(k_{\mu} a / 2\right)$ and $\hat{k}^{2}=\sum_{\mu} \hat{k}_{\mu}^{2}$. Propagators for other actions can be constructed by inverting the gauge field's linearized equation of motion. It is convenient to be able to vary the choice of gauge to test results.

The fermion and gluon propagators are of course diagonal in color space.

The massless overlap operator is defined so that its eigenvalues lie on a circle of radius $x_{0}$, so

$$
D(0)=x_{0}\left(1+\frac{z}{\sqrt{z^{\dagger} z}}\right)
$$

where $z=d\left(-x_{0}\right) / x_{0}=\left(d-x_{0}\right) / x_{0}$ and $d(m)=d+m$ is the massive Dirac operator for mass $m$ (i.e. $x_{0}$ is equivalent to a negative mass term and $d=i \gamma_{\mu} \rho_{\mu}+\lambda$ as above.) The overall multiplicative factor of $x_{0}$ is a useful convention; when the Dirac operator $d$ is thought of as "small" and Eq. 20 is expanded for small $d, D \simeq d$. Feynman rules for the Wilson overlap action have been given by Ref. [7], and can be straightforwardly be adapted for any kernel action.

For overlap actions, it is customary to define the massive overlap operator in terms of the massless one. as

$$
D\left(m_{q}\right)=\left(1-\frac{m_{q}}{2 x_{0}}\right) D(0)+m_{q} .
$$

This results in an annoying entanglement of the mass with the vertices, complicating a direct computation of the running mass. Fortunately, we can compute the multiplicative renormalization factor for the fermion mass indirectly as the inverse of the scalar current renormalization factor, and we can evaluate the latter expression at zero quark mass. 

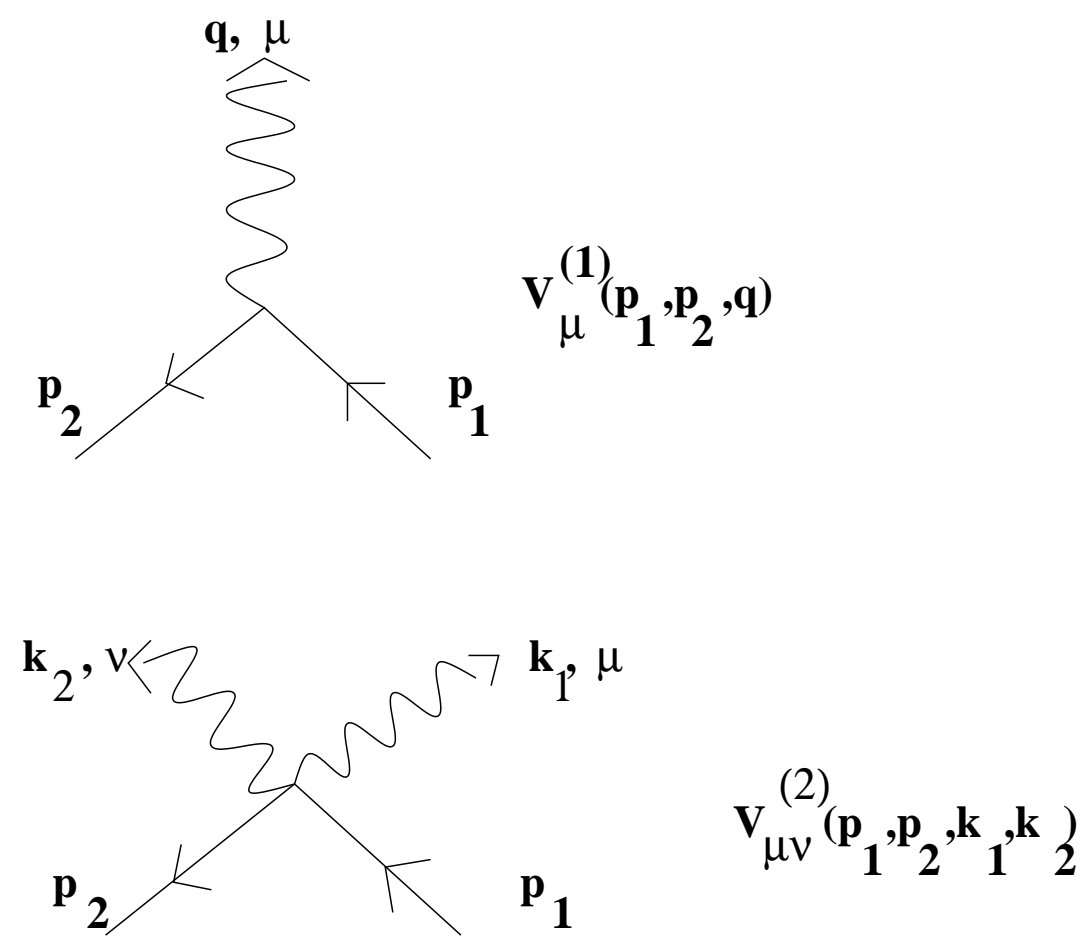

FIG. 1. Three-point and four-point vertices, showing my convention for momentum flows.

\section{Unitary Fat Links}

We will be concerned only with unitary fat links, gauge connections which are themselves elements of the gauge group, even though they may be built of sums of products of the original thin links of the simulation. For smooth fields the fat links have an expansion $V_{\mu}(x)=1+i a B_{\mu}(x)+\ldots$ and the original thin links have an expansion $U_{\mu}(x)=1+i a A_{\mu}(x)+\ldots$. For computations of 2- and 4-quark operator renormalization/matching constants at one loop, only the linear part of the relation between fat and thin links is needed, and it can be parameterized as

$$
B_{\mu}(x)=\sum_{y, \nu} h_{\mu \nu}(y) A_{\nu}(x+y) .
$$

Quadratic terms in (22), which would only be relevant for tadpole graphs, appear as commutators and therefore do not contribute, since tadpoles are symmetric in the two gluons [18,12-14]. In momentum space, the convolution of Eq. (22) becomes a form factor

$$
B_{\mu}(q)=\sum_{\nu} \tilde{h}_{\mu \nu}(q) A_{\nu}(q)
$$

The reader could think of fat-link action Feynman rules as being constructed in two levels: First find the vertices for actions with ordinary thin links, and then replace the thin link by a unitary fat link. Each quark-gluon vertex gets a form factor $h_{\mu \nu}(q)$, where $q$ is the gluon momentum. If all gluon lines start and end on fermion lines, then, effectively, the gluon propagator changes into $G_{\mu \nu} \longrightarrow \tilde{h}_{\mu \lambda} G_{\lambda \sigma} \tilde{h}_{\sigma \nu}$.

(Notice that this is a perturbative realization of the statement that fat link fermion connections can be converted into thin link fermion connections by redefining the fat link variable as an ordinary thin link variable, but with a more complicated pure gauge action.) 


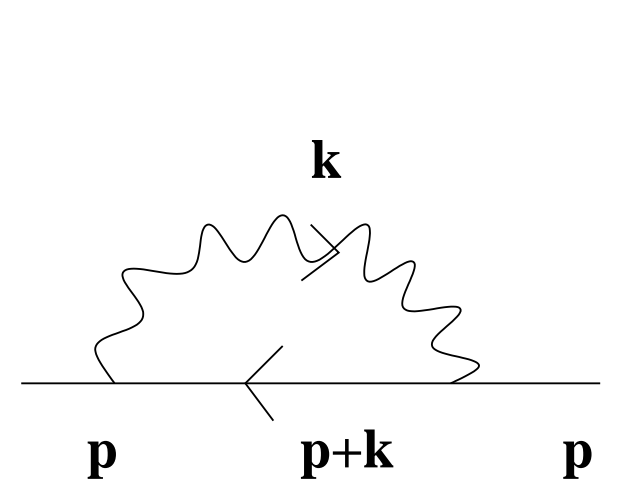

(a)

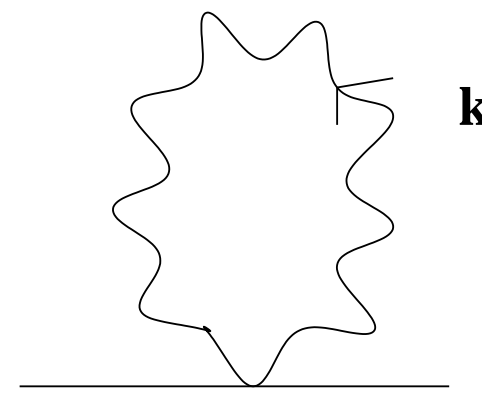

p

(b)

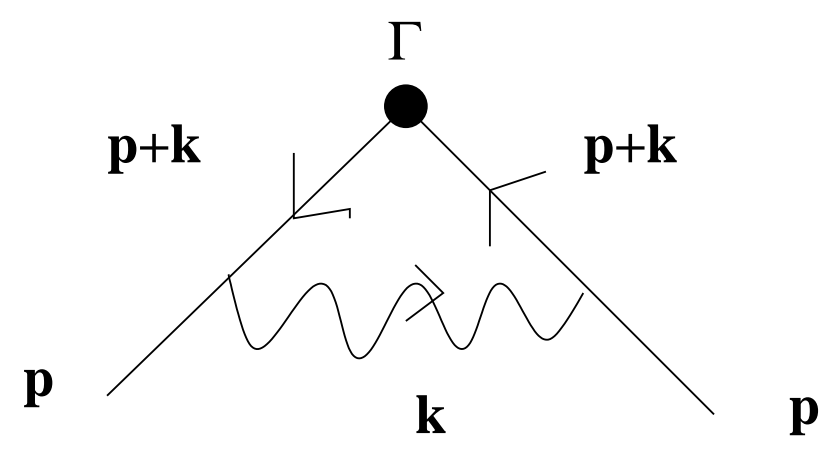

(c)

FIG. 2. The three one-loop diagrams (a) "sunset" and (b) "tadpole" fermion self energy and (c) vertex renormalization, showing my convention for momentum flows.

Form factors for APE and HYP-blocked links are given in Appendix B.

\section{One-loop Diagrams}

The "standard method" for carrying out a lattice perturbation theory calculation is to construct the integrand $I(k, p, m, \lambda, a)$ analytically as a combination of terms multiplying Dirac matrices, and then to project out the desired Dirac structure, producing a single scalar expression $\hat{I}(k, p, m, \lambda, a)$. Possible divergent terms are subtracted out, and the integral is performed using a Monte Carlo routine like VEGAS [20]. The lattice actions I have studied are rather complicated (as are most improved actions) and this procedure appears at first sight to be somewhat daunting. The difficult part of the calculation is the Dirac reduction, particularly as the action (and its Feynman rules) become complicated. One can, of course, do the Dirac algebra using a symbolic manipulation code. However, there is a simpler path for the typical lattice practitioner: Take the parts of the programs which one has already written to do full-scale numerical simulations of the lattice action, and extract the routines which multiply Dirac matrices times spinors. Write the vertices and propagators as explicit $4 \times 4$ matrices, string the expressions together, a and let the computer do all the Dirac multiplication and projection as if it were doing a standard lattice Monte Carlo calculation. All calculations factorize into a set of routines for each action of interest, and a set of integrands (with appropriate projection algorithms) for each coefficient.

This "method" is computationally inefficient, but it is easy to study a wide variety of lattice actions.

I will parameterize the orientation of fermion and gluon momenta through the vertices as shown in Fig. 1.

The fermion self energy is parameterized for small fermion momentum $p$ and small mass $m$ as

$$
\Sigma(p, m)=\Sigma_{0}+i \gamma \cdot p \Sigma_{1}+m \Sigma_{2} .
$$


If nonzero, $\Sigma_{0}$ is (minus) the additive mass renormalization. $\Sigma_{1}$ is the wave-function renormalization, needed for all external lines in vertex functions. The translation of the quark mass from lattice to $\overline{M S}$ regularization $\left(Z_{m}\right)$ is proportional to the difference $\Sigma_{1}-\Sigma_{2}$. While $\Sigma_{0}$ is finite (in lattice units; it is proportional to $1 / a$ and so diverges in the continuum limit), $\Sigma_{1}$ and $\Sigma_{2}$ are infared divergent.

$\Sigma$ itself is a sum of two terms, as shown in Fig. 2. The "sunset" graph, Fig. 2(a), uses first order vertices:

$$
\Sigma_{a}=g^{2} \int_{k} V_{\mu}^{(1)}(p, p+k,-k) S(p+k) V_{\nu}^{(1)}(p+k, p, k) G_{\mu \nu}(k) .
$$

The "tadpole" graph, Fig. 2(b), is

$$
\Sigma_{b}=-\frac{g^{2}}{2} \int_{k} V_{\mu \nu}^{(2)}(p, p, k,-k) G_{\mu \nu}(k) .
$$

Momenta are labeled as shown in the figure. Recall that we usually set $p=0$.

To extract $\Sigma_{1}$ and $\Sigma_{2}$, I expand the propagators and vertices in a power series in $p$ and $m$ (respectively) and keep the leading term. If the free Dirac operator is of the form of Eq. (18) this is straightforward to do. I have performed this expansion analytically, since these two quantities are IR divergent, and require subtraction. Below, the quantity $S_{1}$ will be used, with where $\Sigma_{1}=g^{2} C_{F} /\left(16 \pi^{2}\right) S_{1}$.

The renormalization of currents involves both the vertex graph and $\Sigma_{1}$. I compute the vertex graph simply by taking the amplitude

$$
\left.V^{\Gamma}=\int_{k} V_{\mu}^{(1)}(p, p+k,-k)\right] S(p+k) \Gamma\left[S(p+k) V_{\nu}^{(1)}(p+k, p, k)\right] G_{\mu \nu}(k),
$$

evaluating it at $p=0, m=0$ (with gluon mass $\lambda$ ) and tracing it with the appropriate Dirac projector, before doing the integral. For the vector and axial currents, I average over traces in the four cardinal dimensions.

An alternate parameterization of the vertex allows a connection to matching coefficients of the four fermion operators of the effective field theory of electroweak interactions: Write

$$
V^{\Gamma}=K_{0} \Gamma+K_{1} \gamma_{\mu} \Gamma \gamma_{\mu}+K_{2} \gamma_{\mu} \gamma_{\nu} \Gamma \gamma_{\nu} \gamma_{\mu}+\ldots
$$

Grouping propagator-vertex products,

$$
V^{\Gamma}=\int_{k} T^{1}(p, k) \Gamma T^{2}(p, k) G_{\mu \nu}(k),
$$

with $T^{1}(p, k)=V_{\mu}^{(1)}(p, p+k,-k) S(p+k)$ and $T^{2}(p, k)=S(p+k) V_{\mu}^{(1)}(p, p+k,-k)$. One can find the $K$ 's by projecting the $T$ 's onto elements of the Clifford algebra, $T=T_{0}+\gamma_{\mu} T_{1}+\sigma_{\mu \nu} T_{2}+\ldots$, with $T_{0}=1 / 4 \operatorname{Tr} T, T_{1}=1 / 4 \operatorname{Tr} \gamma_{\mu} T$, and

$$
T_{2}=1 / 8 \sum_{\mu \neq \nu} \operatorname{Tr}\left(\gamma_{\mu} \gamma_{\nu}-\gamma_{\nu} \gamma_{\mu}\right) T
$$

Direct computation plus a consideration of lattice symmetries then allows us to extract the separate terms of Eq. (28), (here in Feynamn gauge) as

$$
K_{0}=\int_{k}\left(T_{0}^{1} T_{0}^{2}-2 T_{2}^{1} T_{2}^{2}\right) G_{\mu \mu}
$$

and

$$
K_{2}=\int_{k} \frac{1}{2} T_{2}^{1} T_{2}^{2} G_{\mu \mu} .
$$

The Wilson and clover actions have only the listed terms $\left(K_{0}, K_{1}, K_{2}\right)$. The overlap action only has nonzero $K_{0}$ and $K_{2}$ terms. The continuum calculation with massless fermions only has nonzero $K_{2}$. Finally, actions which only approximate an overlap action could in principle span the Clifford algebra, although the coefficients of the other terms would be small if the action were a good approximation. This happens for the non-overlap planar action with HYP links. 
The $K_{1}$ term makes its presence felt most malignantly in the one loop correction to four fermion operators, where it is responsible for "bad" operator mixing into opposite-chirality operators. It poisons lattice calculations of $B_{K}$ with Wilson-type quarks.

To find the full Z-factor, and $q^{*}$, we also need the coefficients of $J_{1}$ and $J_{2}$ (in the notation of Eq. 13). These are recorded in Table I. These results are certainly not new, but it is useful to collect them.

If only $K_{0}, K_{1}$, and $K_{2}$ are nonzero, we can immediately write down relations (which appear many times in the literature) between the lattice parts of the matching coefficients of the scalar $(\mathrm{S})$, pseudoscalar $(\mathrm{P})$, vector $(\mathrm{V})$, axial vector (A), and tensor (T) currents: The only one we will need below is

$$
I_{S}-I_{P}=2\left(I_{V}-I_{A}\right)=8 K_{1} .
$$

These relations can be used to relate the matching factors for four-fermion operators to those for bilinears. The earliest reference I can find for this decomposition is by Martinelli [21] and it has been written down most usefully by Gupta, Bhattacharya, and Sharpe [15]. A complication which arises in this case is the prescription used to define $\gamma_{5}$ away from four dimensions. The combination of $1 / \epsilon$ factors from integrals related to Eq. 2 and $\epsilon$ factors from the Dirac algebra is different in the bilinear and four-fermion cases, meaning that a particular four-fermion $Z-$ factor into a particular continuum convention is a linear combinations of bilinear $Z_{i}$ 's plus extra constant terms.

I found it most straightforward way to find these constants was to do the continuum Dirac algebra using the techniques of Ref. [22] (basically copying the examples of Ref. [23]). In order to extract the momentum scale $q^{*}$, we need to separate the $A$ and $B$ coefficients of Eq. 2. Most lattice calculations do not include penguin graphs, and four operators are needed for the most frequently performed four fermion matrix elements, combinations of

$$
O=\left(\bar{q}_{\alpha}^{(1)} \Gamma_{1} q_{\beta}^{(2)}\right) \otimes\left(\bar{q}_{\gamma}^{(3)} \Gamma_{2} \hat{q}_{\delta}^{(4)}\right) .
$$

Special cases are (a) $\Gamma_{1}=\Gamma_{2}=\gamma_{\mu}\left(1-\gamma_{5}\right)$ : if color labels $\alpha=\delta, \beta=\gamma, O=O_{1}$; if $\alpha=\beta, \gamma=\delta, O=O_{2}$; and (b)the isospin $3 / 2$ operators for electroweak penguins,

$$
O_{7}^{3 / 2}=\left(\bar{s}_{\alpha} \gamma_{\mu}\left(1-\gamma_{5}\right) d_{\alpha}\right)\left[\left(\bar{u}_{\beta} \gamma_{\mu}\left(1+\gamma_{5}\right) u_{\beta}\right)-\left(\bar{d}_{\beta} \gamma_{\mu}\left(1+\gamma_{5}\right) d_{\beta}\right)\right]+\left(\bar{s}_{\alpha} \gamma_{\mu}\left(1-\gamma_{5}\right) u_{\alpha}\right)\left(\bar{u}_{\beta} \gamma_{\mu}\left(1+\gamma_{5}\right) d_{\beta}\right)
$$

and

$$
O_{8}^{3 / 2}=\left(\bar{s}_{\alpha} \gamma_{\mu}\left(1-\gamma_{5}\right) d_{\beta}\right)\left[\left(\bar{u}_{\beta} \gamma_{\mu}\left(1+\gamma_{5}\right) u_{\alpha}\right)-\left(\bar{d}_{\beta} \gamma_{\mu}\left(1+\gamma_{5}\right) d_{\alpha}\right)\right]+\left(\bar{s}_{\alpha} \gamma_{\mu}\left(1-\gamma_{5}\right) u_{\beta}\right)\left(\bar{u}_{\beta} \gamma_{\mu}\left(1+\gamma_{5}\right) d_{\alpha}\right) .
$$

Ingredients for the non-penguin mixing factors are given in Table II.

\section{SOME EXAMPLES}

I have studied a large variety of lattice actions with thin and fat links. Numerical integrals are checked, when possible, by comparison against published results. For currents, I vary the gauge choice $\xi$ and check that integrals (and integrands) remain gauge invariant. As a general rule using double precision insures that the integrands are gauge invariant point by point to a few parts in $10^{5}$.

My results for any standard thin link action (Wilson, clover, Wilson overlap, ...) are (with one exception) not new, and there is no point in republishing old results already in the literature. Results for a standard action with HYP links are mostly unpublished, so I will show them in tables. I will show results for the clover and planar actions, since the HYP clover action might be an attractive action for simulations. I will also tabulate results for the planar action, with thin and HYP-blocked links, and the HYP-blocked planar overlap.

HYP blocking is characterized by three parameters with "preferred" values $\alpha_{1}=0.75, \alpha_{2}=0.6, \alpha_{3}=0.3$. While HYP blocking is typically presented (and used) at this specific value of these coefficients, it is very useful to show how the matching coefficients vary with the degree of fattening of the link. I will do this simply by multiplying the standard HYP coefficients by an overall scale factor, and tune the scale factor from zero (corresponding to a thin link) to 1 to 1.5. There are many other ways to tune HYP blocking, of course.

With HYP smearing, the first-order formula Eq. (7) is small because of cancellations of negative and positive contributions in the integral, and the second order formula is often needed. The reader will note many "cusps" in the $q^{*}$ plots for HYP actions as I switch from first to second order $q^{*}$ 's. In contrast, as a general rule, under increased APE-smearing $q^{*}$ usually falls slowly towards zero, and the first-order formula for $q^{*}$ works well, unless the actual matrix element vanishes. 


\section{A. Additive mass renormalization}

All of the features of fat link perturbation theory can be seen in the additive mass renormalization for non-overlap actions. Fig. 3 shows the additive mass renormalization for thin link Wilson and clover fermions, with $c_{S W}=1$, and for the planar action with HYP-blocked links. The graphs show $S_{0}$, with the definition $\delta m=\alpha_{s}\left(q^{*}\right) S_{0}$. We see that all these thin link actions have large additive mass renormalization. The addition of the clover term reduces $S_{0}$ by about half, but it is still big. Smearing the gauge fields has a dramatic effect on $S_{0}$, until the scale factor for HYP blocking exceeds unity. At this point the blocking enhances the large gluon momentum region in the integrand rather than suppressing it. This effect shows little dependence on the choice of fermion action (planar or clover).
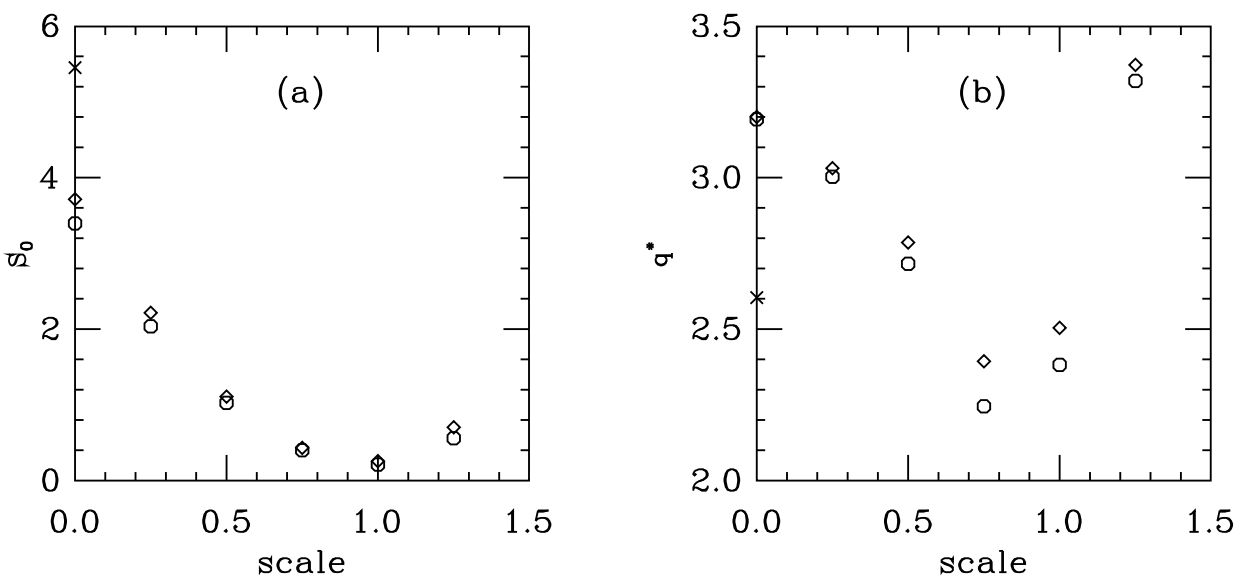

FIG. 3. (a) $S_{0}$ parameterizing additive mass renormalization for thin link Wilson (cross), and HYP-link link clover (diamond) and planar (octagons) fermions and (b) their momentum scale $q^{*}$ with HYP blocking, with the optimum parameters scaled by the shown overall scale factor.

\section{B. Fermion Bilinears}

Next we turn to results for fermion bilinears, parameterized as $Z_{i}=1+z_{i} g^{2}\left(q^{*}\right) C_{F} /\left(16 \pi^{2}\right)$. Table III shows Z-factors for currents for the thin link and HYP-blocked planar action. The values quoted in all tables have an uncertainty smaller than \pm 1 in their rightmost digit.

Table IV shows Z-factors for the thin link and HYP-blocked naive fermions. These results form a (tiny) subset of an extensive calculation of matching factors for staggered fermions by Sharpe and W. Lee [13]. The $q^{*}$ values are new.

Matching factors and $q^{*}$ scales for the local vector and axial vector currents for clover fermions and planar fermions as a function of fattening strength are shown in Figs. 4 and 5, and results for the local scalar and pseudoscalar currents are show in in Figs. 6 and 7. The qualitative features of fattening are the same for both actions: for thin link actions all $z$ 's are large in magnitude and the differences between "chiral partners" (such as $z_{V}$ and $z_{A}$ ) are also large. Either HYP action has tiny z's (order unity) with differences an order of magnitude smaller. 

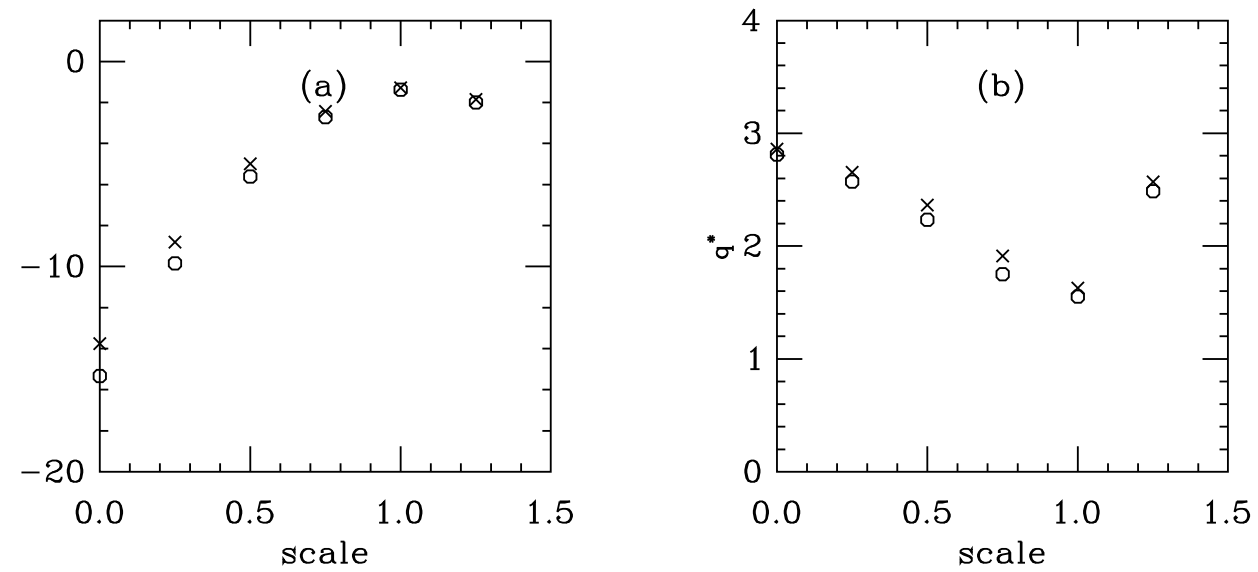

FIG. 4. (a) $z_{V}$ and $z_{A}$, the coefficients of $g^{2} C_{f} /\left(16 \pi^{2}\right)$ for the local vector (octagons) and axial current renormalization constants (crosses) for clover fermions with HYP blocking. (b) Momentum scale $q^{*}$ for the local vector and axial currents.
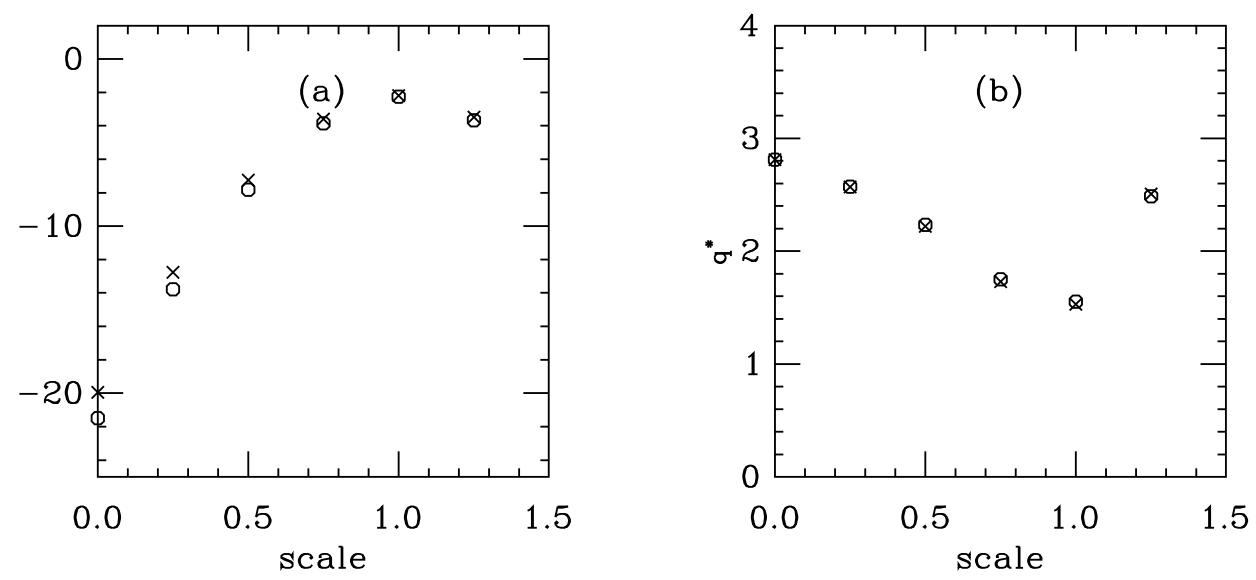

FIG. 5. (a) $z_{V}$ and $z_{A}$ and their $q^{*}$ 's, for the HYP-planar action, labelled as in Fig. 4.
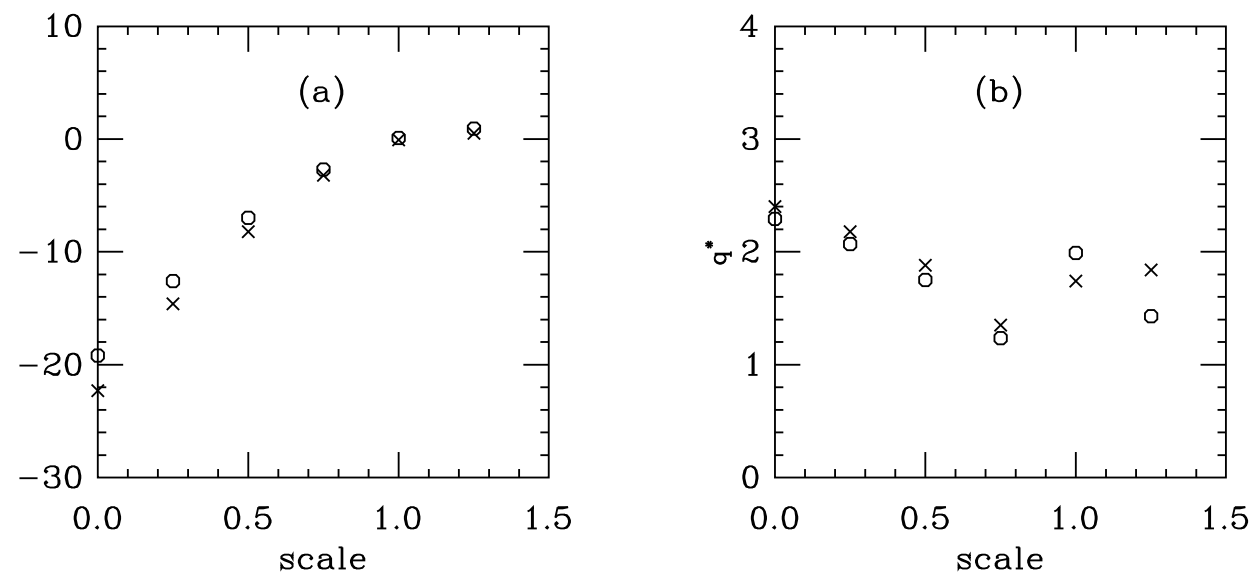

FIG. 6. (a) $z_{S}$ and $z_{P}$, the coefficients of $g^{2} C_{F} /\left(16 \pi^{2}\right)$ for the local scalar and pseudoscalar current renormalization constants (evaluated at $\mu a=1$ ) for clover fermions, labelled by octagons and crosses, respectively. (b) Momentum scale $q^{*}$. 

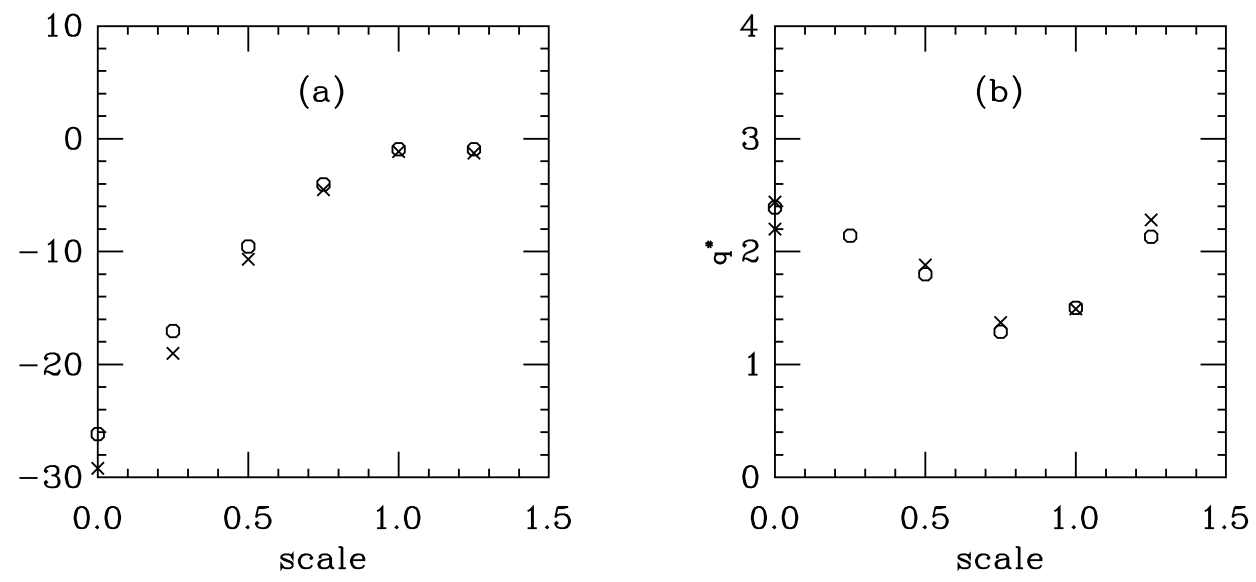

FIG. 7. (a) $z_{S}$ and $z_{P}$ and their $q^{*}$ 's, for HYP-planar action, labelled as in Fig. 6.

Results for overlap actions parallel those for nonchiral actions: matching factors drop when the clover term is included, and drop more when the links are fattened. To illustrate this, I present results for the thin link Wilson and clover overlap, both with $x_{0}=1.6$, and for the HYP planar overlap, in Figs. 8 and 9. A table of results for the HYP-planar overlap is given in Table V.
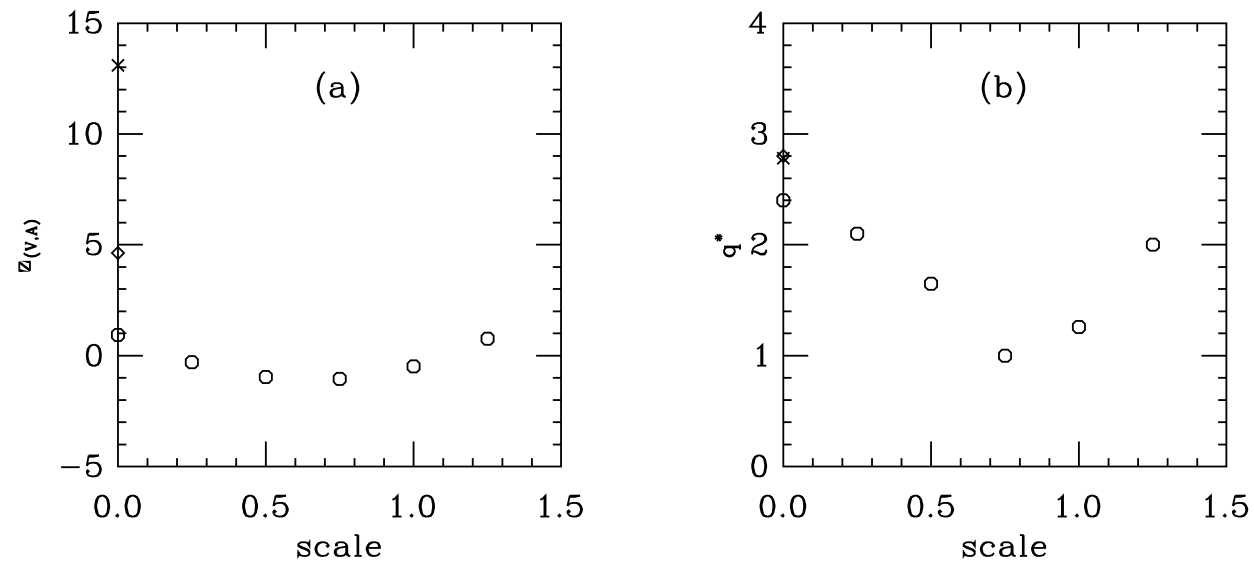

FIG. 8. Coefficient of $g^{2} C_{F} /\left(16 \pi^{2}\right)$ (a) and momentum scale $q^{*}$ (b) for the local vector (and axial vector) currents for overlap fermions with the planar action kernel and HYP links, at $x_{0}=1.6$. Octagons label the planar action; also shown are the thin link Wilson (cross) and clover (diamond) actions.
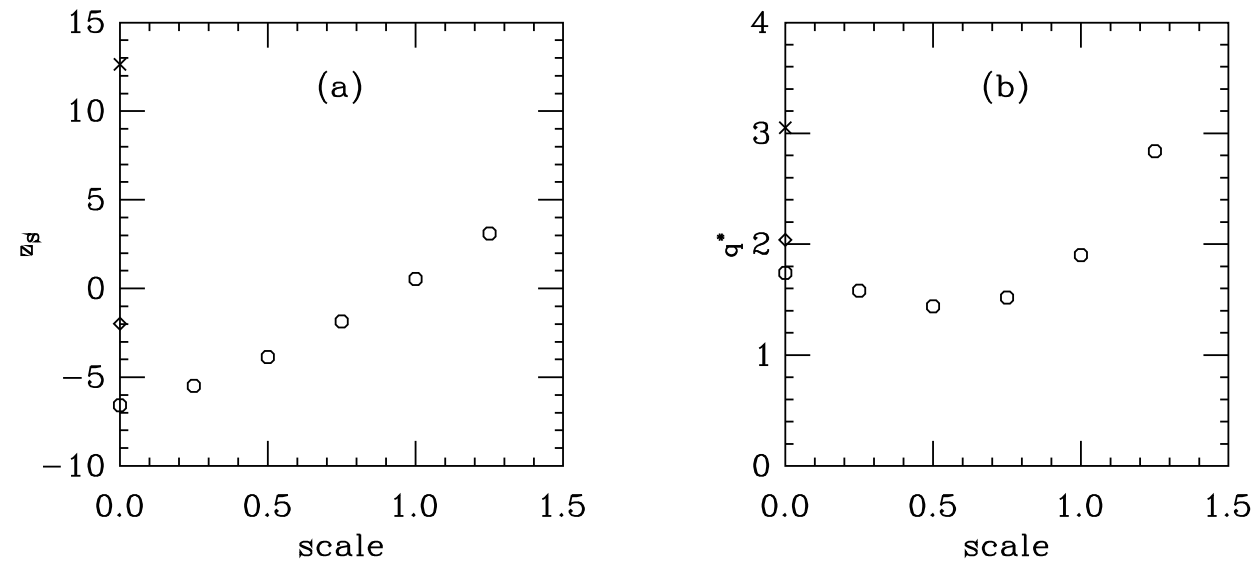
FIG. 9. Coefficient of $g^{2} C_{F} /\left(16 \pi^{2}\right)$ (a) and momentum scale $q^{*}$ for the local scalar (and pseudoscalar) currents for overlap fermions with the planar action kernel and HYP links, at $x_{0}=1.6$. Octagons label the planar action; also shown are the thin link Wilson (cross) and clover (diamond) actions.

\section{Four fermion operators}

Some sample results for four fermion operators are shown in Table VI. My results for (ordinary non-overlap) Wilson fermions agree with the DRED(EZ) results of Ref. [24] and the NDR results of Ref. [15]. My Wilson overlap results satisfy the connection between $Z_{+}, Z_{V, A}$, and $Z_{P, S}$ of Ref. [15] and Table II. For the special case of radius $x_{0}=1$ they agree with a calculation of P. Weisz [25]. They differ by an overall additive factor of $14 / 3$ (the precise value comes from Weisz; I have only determined this factor numerically) from the results of Ref. [9]. NDR four fermion matching coefficients for the Wilson overlap action for many radii can readily be constructed from any desired operator using the tables of bilinears from Ref. [8] and the results of Ref. [15] or Table II (though finding the $q^{*}$ scale will require actually doing some integrals).
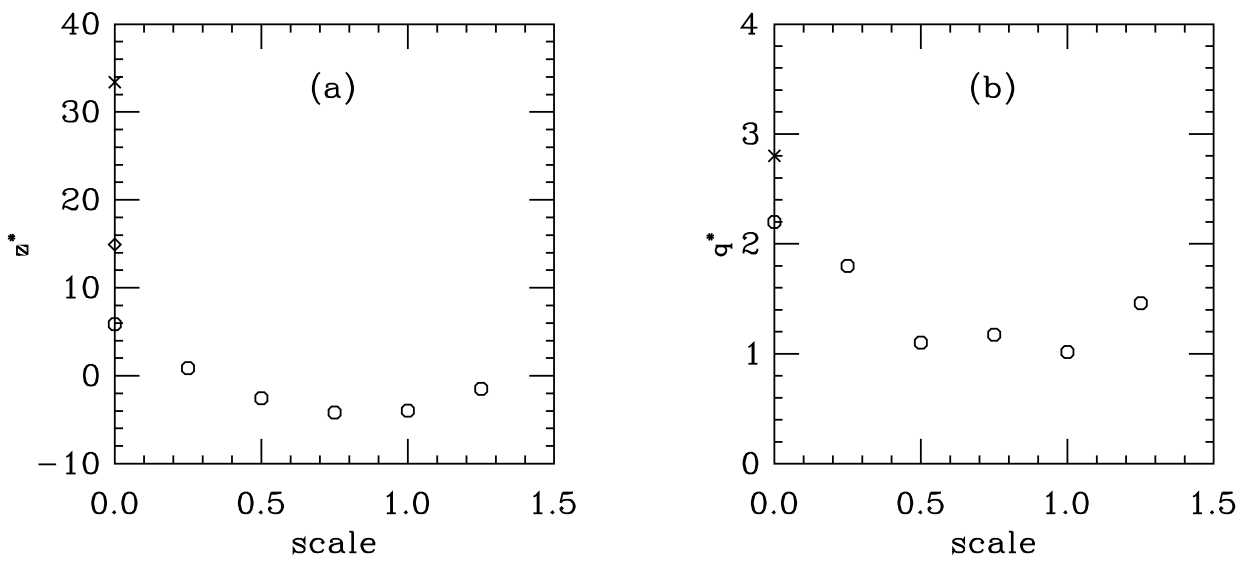

FIG. 10. (a) Coefficient of $g^{2} /\left(16 \pi^{2}\right)$ at scale $\mu a=1$ and (b) momentum scale $q^{*}$ for $Z_{+}$for matching lattice and NDR overlap fermions with the planar action kernel, with $x_{0}=1.6$. Octagons label the planar action; also shown are the thin link Wilson (cross) and clover (diamond) actions.

In Fig. 10 I show results for the HYP planar action kernel, as well as for the thin link Wilson and clover overlaps. In all cases I set $x_{0}=1.6$. There is a large reduction in $z_{+}$by converting from a Wilson kernel to a clover kernel even without fat links (recall that the planar action includes a clover term). Fattening the links further reduces $z_{+}$.
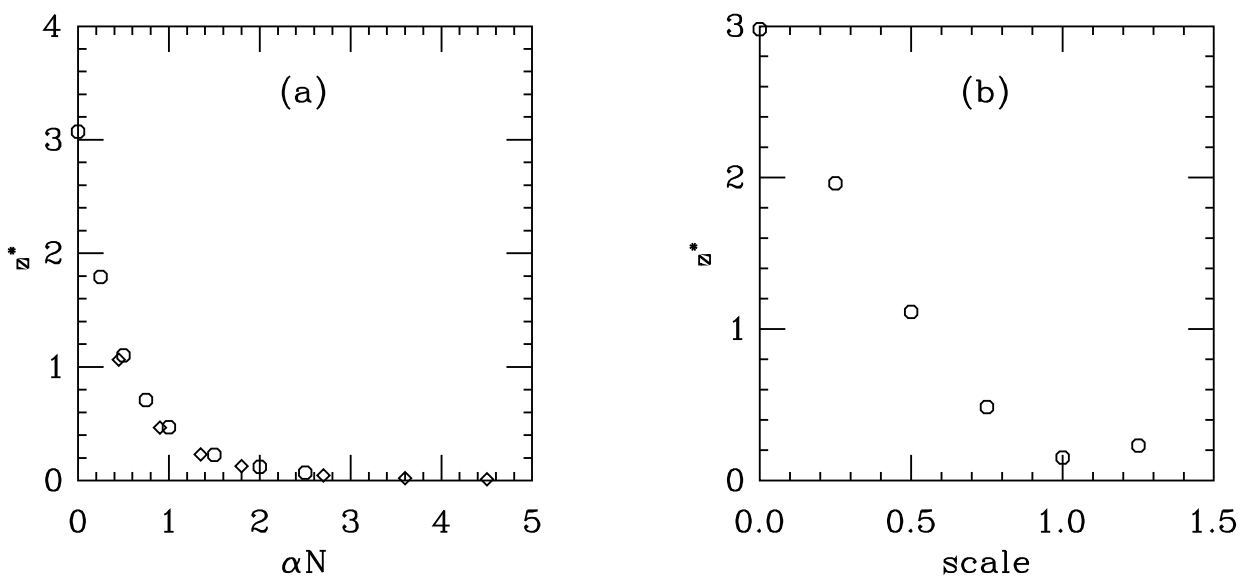

FIG. 11. $Z^{*}$, the wrong-chirality mixing amplitude, for clover fermions with (a) APE blocking (octagons for $\alpha=0.25$ and diamonds for $\alpha=0.45$ ) (b) HYP blocking, with the optimum parameters scaled by the shown overall scale factor. 
Finally, to return to non-chiral actions, we can ask how fattening alters the mixing into different chiral sectors. This quantity is parameterized by the coefficient $Z^{*}=-8 K_{1}$. From Eq. $28, Z^{*}=z_{V}-z_{A}=2\left(z_{P}-z_{S}\right)$. As we have already seen, fattening pushes all the $z$ 's closer to zero, and so their differences also become small. Values of $Z^{*}$ for the clover action with APE blocking and HYP blocking are shown in Fig. 11. As expected, either smearing can cut $Z^{*}$ by over an order of magnitude.

The Wilson thin link fermion action value $Z^{*}=9.6[24]$ is much greater than even the thin-link clover result. Converting to fat links without also turning on the clover term helps, but will not be productive: for example, with scale factor unity, the HYP-blocked Wilson action has $Z^{*}=2.29$, while including a $c_{S W}=1$ clover term cuts this number to 0.16 .

Results for the operators $O_{7}$ and $O_{8}$ for the Wilson and planar overlaps are shown in Table VII.

Let's use these results to consider a numerical example of the ambiguities which will afflict our calculation of mixing. In all cases we will match the lattice and NDR calculations at a scale $q_{i}$ and run the NDR result to $q_{f}=2 \mathrm{GeV}$ using the two-loop evolution equation. We will suppose we are using the Wilson gauge action a coupling at $\beta=5.9$ and assume that the inverse lattice spacing is $1 / a=1.58 \mathrm{GeV}$. (These are typical numbers from simulations [28]). A standard calculation a la Ref. [5] beginning with the logarithm of the average plaquette yields $\alpha \overline{\overline{M S}}(q a=1)=0.199$ and $\alpha \overline{M S}(q a=\pi)=0.137$. We begin with the HYP-planar overlap action and imagine just matching at a scale $q_{1}=1 / a$ or $\pi / a$, and running up. Then the full matching and running matrix is

$$
Z\left(q_{f}, q_{i}\right) Z\left(q_{i}\right)=\left(\begin{array}{cc}
0.979 & -0.006 \\
-0.039 & 1.099
\end{array}\right)
$$

and the result at $q_{i}=\pi / a$ is

$$
Z\left(q_{f}, q_{i}\right) Z\left(q_{i}\right)=\left(\begin{array}{cc}
0.998 & -0.030 \\
-0.043 & 1.012
\end{array}\right)
$$

Next, we compute a "lowest order" $q^{*}$ from Eq. 16 . We have $q_{1}^{*} a=3.6, q_{2}^{*} a=0.68$, and

$$
Z\left(q_{f}, q_{i}\right) Z\left(q_{i}\right)=\left(\begin{array}{cc}
0.966 & -0.020 \\
-0.009 & 1.112
\end{array}\right)
$$

Notice that in all cases the matrices are nearly diagonal.

In contrast, the first choice for the Wilson action (just set $q^{*} a=1$ )) gives

$$
Z\left(q_{f}, q_{i}\right) Z\left(q_{i}\right)=\left(\begin{array}{cc}
1.558 & -0.017 \\
-0.033 & 1.640
\end{array}\right)
$$

matching at $q^{*} a=\pi$ and running gives

$$
Z\left(q_{f}, q_{i}\right) Z\left(q_{i}\right)=\left(\begin{array}{cc}
1.412 & -0.071 \\
-0.050 & 1.304
\end{array}\right)
$$

while using Eq. (16), with $q^{*} a=(3.0,2.3)$, we have

$$
Z\left(q_{f}, q_{i}\right) Z\left(q_{i}\right)=\left(\begin{array}{cc}
1.459 & -0.016 \\
-0.042 & 1.530
\end{array}\right)
$$

The off diagonal terms in $Z$ are small and not too dependent on the running scheme, but the diagonal coupling of the operators suffers a giant renormalization. One probably should treat perturbation theory results for the Wilson overlap cautiously for these factors.

\section{CONCLUSIONS}

Fattening the gauge connections of a lattice fermion action is a simple way to reduce the size of perturbative matching coefficients. HYP blocking is a particularly felicitous choice: it combines large scale smoothing with locality. It is clear from the results presented that the qualitative features of fattening do not depend on the specific choice of parameters. In situations where full chiral symmetry might not be necessary, the HYP-blocked clover action might be an attractive choice for a light quark action. 
In this work I have only considered lattice actions with scalar and vector couplings and nearest and next-nearest couplings. It would be easy to construct the Feynman rules for "hypercubic" actions (such as those of Refs. [19] or [26]). Techniques similar to the ones used here could be applied (with only a little more effort) to the more complicated approximate fixed point or overlap actions used by several other authors, whose kernels fill out the entire Clifford algebra [27].

The result for $Z_{+}$will be used in a lattice calculation of $B_{K}$ using the HYP-blocked planar overlap [28].

\section{ACKNOWLEDGMENTS}

I would like to thank Anna Hasenfratz, Roland Hoffman, and Francesco Knechtil for many discussions about HYP blocking, and Weonjong Lee, Steve Sharpe, and Peter Weisz for correspondence. I am very much indebted to Claude Bernard for introducing me to lattice perturbation theory. This work was supported by the U. S. Department of Energy with grant DE-FG03-95ER40894.

\section{APPENDIX A: FEYNMAN RULES FOR PLANAR-ACTION FERMIONS}

The non-overlap fermion action I am most interested in has scalar and vector couplings to fermions offset on nearest-neighbor and diagonal-offset sites, and minimal length gauge paths built of unitarized fat links connecting them. The nearest-neighbor vector and scalar couplings are labeled $\rho_{1}$ and $\lambda_{1}$; the diagonal $(\vec{r}= \pm \hat{\mu} \pm \hat{\nu}, \nu \neq \mu)$ couplings are $\rho_{2}$ and $\lambda_{2}$. There is also a local scalar coupling $\lambda_{0}$. For the Wilson or clover action, $\rho_{2}=\lambda_{2}=0$; $\rho_{1}=\lambda_{1}=-1 / 2$. For the "planar" action of Ref. [2] $\lambda_{1}=-0.170, \rho_{\mu}^{(1)}=-0.177$ and diagonal neighbors $(\vec{r}= \pm \hat{\mu} \pm \hat{\nu}$, $\nu \neq \mu ; \lambda_{2}=-0.061, \rho_{\mu}^{(2)}=\rho_{\nu}^{(2)}=-0.0538$. The constraint $\lambda_{=}-8 \lambda_{1}-24 \lambda_{2}$ enforces masslessness on the free spectrum, and $-1=2 \rho_{\mu}^{(1)}+12 \rho_{\mu}^{(2)}$ normalizes the action to $-\bar{\psi} i \gamma_{\mu} \partial_{\mu} \psi$ in the naive continuum limit.

The free fermion action is then

$$
d(p)=\lambda_{0}+2 \lambda_{1} \sum_{\mu} \cos p_{\mu}+4 \lambda_{2} \sum_{\mu, \nu<\mu} \cos q_{\mu} \cos q_{\nu}+i \sum_{\mu} \gamma_{\mu} \sin q_{\mu}\left(2 \rho_{1}+4 \rho_{2} \sum_{\nu \neq \mu} \cos q_{\nu}\right) .
$$

Nearest-neighbor connections basically contribute rescaled versions of the usual Wilson-action Feynamn rules. The diagonal-offset neighbor gauge connections are taken to be an average of the two length-two shortest paths connecting the fermions (each of which is a product of unitarized HYP links). Neglecting the form factor arising from fattening, the first order vertex $V_{\mu}^{(1)}$ is

$$
\begin{aligned}
V_{\mu}^{(1,)}=2 i \gamma_{\mu}\left\{\left(\rho_{1}+\rho_{2} \sum_{\nu \neq \mu}\left(\cos p_{1}+\cos p_{2}\right)_{\nu}\right) \cos \left(\frac{\left(p_{1}+p_{2}\right)_{\mu}}{2}\right\}\right. \\
+\sum_{\nu \neq \mu} \gamma_{\nu}\left(2 i \rho_{2} \sin \left(\frac{\left(p_{1}+p_{2}\right)_{\mu}}{2}\right)\left(\sin p_{1 \nu}+\sin p_{2 \nu}\right)\right) \\
-2\left(\lambda_{1}+\lambda_{2} \sum_{\nu \neq \mu}\left(\cos p_{1}+\cos p_{2}\right)_{\nu}\right) \sin \left(\frac{\left(p_{1}+p_{2}\right)_{\mu}}{2}\right) .
\end{aligned}
$$

I also include a clover term in $V^{(1)}$ :

$$
V_{\mu}^{(1, c l)}=-\frac{1}{2} C_{S W} \gamma_{\mu} \sum_{\nu \neq \mu} \gamma_{\nu} \sin k_{\nu} \cos \frac{k_{\mu}}{2}
$$

(My definition of $C_{S W}$ would be unconventional for Wilson fermions if $\lambda_{1} \neq 1 / 2$, or $r \neq 1$ in usual usage.) The planar action has $C_{S W}=1.03$.

The expression for the four-point vertex is long, but in all the calculations done here, I only need an expression for the vertex at zero fermion momenta and for its first derivative with respect to (equal) fermion momenta, also at zero momentum. In that limit,

$$
\left.V_{\mu \nu}^{(2)}=\delta_{\mu \nu}\left(-2 \lambda_{1}-12 \lambda_{2}\right)+\left(1-\delta_{\mu \nu}\right) 4 \lambda_{2} \sin \frac{k_{\mu}}{2} \sin \frac{k_{\nu}}{2}\right)
$$

and its derivative at zero external fermion momenta is

$$
\frac{\partial V_{\mu}^{(2)}}{\partial p_{\nu}}=p_{\nu} \gamma_{\nu}\left(\delta_{\mu \nu}\left(-2 \rho_{1}-24 \rho_{2}\right)+\left(1-\delta_{\mu \nu}\right) 4 \rho_{2} \sin \frac{k_{\mu}}{2} \sin \frac{k_{\nu}}{2}\right)
$$




\section{APPENDIX B: EXPLICIT FORMULAS FOR FAT LINKS}

APE blocking: The link after $n+1$ smearings is related to the link after $n$ smearings by

$$
\begin{aligned}
& V_{\mu}^{(n+1)}(x)= \operatorname{Proj}_{S U(3)}\left((1-\alpha) V_{\mu}^{(n)}(x)\right. \\
&+\alpha / 6 \sum_{\nu \neq \mu}\left(V_{\nu}^{(n)}(x) V_{\mu}^{(n)}(x+\hat{\nu}) V_{\nu}^{(n)}(x+\hat{\mu})^{\dagger}\right. \\
&\left.\left.+V_{\nu}^{(n)}(x-\hat{\nu})^{\dagger} V_{\mu}^{(n)}(x-\hat{\nu}) V_{\nu}^{(n)}(x-\hat{\nu}+\hat{\mu})\right)\right) .
\end{aligned}
$$

$V_{\mu}^{(n+1)}(x)$ is projected back onto $S U(3)$ after each step, and $V_{\mu}^{(0)}(n)=U_{\mu}(n)$ is the original link variable. The momentum-space smearing factor for one level of smearing is

$$
\tilde{h}_{\mu \nu}(q)=f(q)\left(\delta_{\mu \nu}-\frac{\hat{q}_{\mu} \hat{q}_{\nu}}{\hat{q}^{2}}\right)+\frac{\hat{q}_{\mu} \hat{q}_{\nu}}{\hat{q}^{2}}
$$

with $\hat{q}_{\mu}=\frac{2}{a} \sin \left(\frac{a q_{\mu}}{2}\right)$ and $f(q)=1-\frac{\alpha}{6} \hat{q}^{2}$. After $N$ smearings, $\tilde{h}_{\mu \nu}(q)$ becomes $\tilde{h}_{\mu \nu}^{N}(q)$, which is just $\tilde{h}_{\mu \nu}$ with $f$ replaced by $f^{N}$.

HYP blocking: The momentum space version of HYP blocking is

$$
\begin{aligned}
B_{\mu}(q) & =\sum_{\nu} h_{\mu \nu}(q) A_{\nu}(q)+O\left(A^{2}\right), \text { where } \\
h_{\mu \nu}(q) & =\delta_{\mu \nu}\left[1-\frac{\alpha_{1}}{6} \sum_{\rho} \hat{q}_{\rho}^{2} \Omega_{\mu \rho}(q)\right]+\frac{\alpha_{1}}{6} \hat{q}_{\mu} \hat{q}_{\nu} \Omega_{\mu \nu}(q), \\
\Omega_{\mu \nu}(q) & =1+\alpha_{2}\left(1+\alpha_{3}\right)-\frac{\alpha_{2}}{4}\left(1+2 \alpha_{3}\right)\left(\hat{q}^{2}-\hat{q}_{\mu}^{2}-\hat{q}_{\nu}^{2}\right)+\frac{\alpha_{2} \alpha_{3}}{4} \prod_{\eta \neq \mu, \nu} \hat{q}_{\eta}^{2} .
\end{aligned}
$$

with $\alpha_{1}=0.75, \alpha_{2}=0.6$, and $\alpha_{3}=0.3$ the favored parameterization of Ref. [3].

\begin{tabular}{lccc}
\hline \hline & $I_{\text {latt }}$ & $\mathrm{A}$ & $\mathrm{B}$ \\
$Z_{V}$ & $4 K_{2}+2 K_{1}+K_{0}-S_{1}$ & 0 & 0 \\
$Z_{A}$ & $4 K_{2}-2 K_{1}+K_{0}-S_{1}$ & 0 & 0 \\
$Z_{P}$ & $16 K_{2}-4 K_{1}+K_{0}-S_{1}$ & 3 & $-1 / 2$ \\
$Z_{S}$ & $16 K_{2}+4 K_{1}+K_{0}-S_{1}$ & 3 & $-1 / 2$ \\
\hline \hline
\end{tabular}

TABLE I. Ingredients for matching coefficients for some local operators.

\begin{tabular}{lccc}
\hline \hline & $I_{\text {latt }}$ & $\mathrm{A}$ & $\mathrm{B}$ \\
$Z_{+}$ & $8 / 3\left(K_{2}+K_{0}-S_{1}\right)$ & -2 & $-5 / 3$ \\
$Z_{-}$ & $80 / 3 K_{2}-8 / 3\left(K_{0}-S_{1}\right)$ & 4 & $10 / 3$ \\
$Z_{77}$ & $4 / 3\left(5 K_{2}+2\left(K_{0}-S_{1}\right)\right)$ & -1 & $7 / 6$ \\
$Z_{88}$ & $4 / 3\left(32 K_{2}+2\left(K_{0}-S_{1}\right)\right)$ & 8 & $-1 / 3$ \\
$Z_{78}$ & $12 K_{2}$ & 3 & $-7 / 2$ \\
$Z_{87}$ & 0 & 0 & -3 \\
\hline \hline
\end{tabular}

TABLE II. Ingredients for the (non-penguin parts) of matching coefficients for some four-fermion operators. 


\begin{tabular}{|c|r|r||r|}
\hline \hline action & process & $z_{i}$ & $q^{*}$ \\
\hline thin planar & & & 3.71 \\
& $S_{0}$ & -21.5 & 3.19 \\
& $Z_{V}$ & -20.0 & 2.8 \\
& $Z_{A}$ & -26.2 & 2.8 \\
& $Z_{S}$ & -29.3 & 2.4 \\
& $Z_{P}$ & 0.26 & 2.4 \\
\hline HYP planar & & -2.27 & 2.37 \\
& $S_{0}$ & -2.19 & 1.55 \\
& $Z_{V}$ & -0.92 & 1.54 \\
& $Z_{A}$ & -1.11 & 1.50 \\
& $Z_{S}$ & $Z_{P}$ & 1.51 \\
& & & \\
\hline \hline
\end{tabular}

TABLE III. Table of Z-factors and $q^{*}$ 's for planar and HYP-planar actions, defined so $Z_{i}=1+z_{i} g^{2} C_{f} /\left(16 \pi^{2}\right) . C_{S W}=1.03$.

\begin{tabular}{|c|r|r|r|r|}
\hline \hline action & process & $z_{i}$ & $q^{*}$ \\
\hline thin & & & 3.27 \\
& $Z_{V, A}$ & -14.8 & 2.79 \\
& $Z_{P, S}$ & -39.2 & 2.36 \\
\hline HYP & & & -0.945 & 2.05 \\
& $Z_{V, A}$ & -0.592 & $Z_{P, S}$ \\
\hline \hline
\end{tabular}

TABLE IV. Table of Z-factors and $q^{*}$ s for thin link and HYP link naive fermion actions.

\begin{tabular}{|c|r|r|}
\hline \hline process & $\mathrm{z}$ & $q^{*}$ \\
\hline$Z_{V, A}$ & -0.489 & 1.26 \\
$Z_{P, S}$ & 0.53 & 1.96 \\
\hline \hline
\end{tabular}

TABLE V. Table of Z-factors and $q^{*}$ 's for bilinears, HYP-planar overlap action.

\begin{tabular}{|c|cc|cc|}
\hline \hline \hline action & $z_{+}$ & $q^{*}$ \\
\hline thin Wilson & & 33.4 & & 2.86 \\
thin Clover & & 14.3 & & 4.07 \\
$0.45 \times 10$ APE Clover & & -5.8 & & 1.05 \\
$0.45 \times 10$ APE planar & & -5.67 & & 0.95 \\
HYP planar & & -3.97 & & 0.92 \\
\hline \hline
\end{tabular}

TABLE VI. Table of Z-factors into NDR and $q^{*}$ 's for $O_{+}=O_{1}+O_{2}$ for overlap actions with various kernels. In all cases $x_{0}=1.6, C_{S W}=1$ for clover action and 1.03 for planar action. $Z_{+}=1+\alpha_{s}\left(q^{*}\right) /(4 \pi)\left(z_{+}+4 \log (a \mu)\right)$. 


\begin{tabular}{|c|c|c|c|c|}
\hline \hline action & $\mathcal{Z}_{77}$ & $\mathcal{Z}_{78}$ & $\mathcal{Z}_{87}$ & \\
\hline thin Wilson $\mathcal{Z}$ & 36.29 & -3.51 & -3 & $\mathcal{Z}_{88}$ \\
thin Wilson $\mathcal{L}_{1}$ & 70.57 & 1.56 & -0.003 & 34.85 \\
thin Wilson $\mathcal{L}_{2}$ & 17.6 & -4.64 & 9.84 & \\
\hline HYP planar $\mathcal{Z}$ & -0.65 & -1.98 & -3 & 19.32 \\
HYP planar $\mathcal{L}_{1}$ & 1.52 & 2.72 & -0.003 & 9.41 \\
HYP planar $\mathcal{L}_{2}$ & 4.94 & -1.65 & 9.69 \\
\hline \hline
\end{tabular}

TABLE VII. Table of Z-factors and $q^{*}$ 's for $O_{7}$ and $O_{8}$ for overlap actions with various kernels. In all cases $x_{0}=1.6$, $C_{S W}=1.03$ for the planar action. $Z_{i j}=\delta_{i j}+\alpha_{s}\left(q^{*}\right) /(4 \pi) \mathcal{Z}_{i j} . \mathcal{L}_{1}$ and $\mathcal{L}_{2}$ are the integrals of $\mathcal{Z}$, weighted by log $q^{2}$ and $\log ^{2} q^{2}$, respectively.

[1] H. Neuberger, Phys. Lett. B417, 141 (1998) [hep-lat/9707022], Phys. Rev. Lett. 81, 4060 (1998) [hep-lat/9806025].

[2] T. DeGrand [MILC collaboration], Phys. Rev. D 63, 034503 (2001) [hep-lat/0007046].

[3] A. Hasenfratz and F. Knechtli, Phys. Rev. D 64, 034504 (2001) [arXiv:hep-lat/0103029]; A. Hasenfratz, R. Hoffmann and F. Knechtli, Nucl. Phys. Proc. Suppl. 106, 418 (2002) [arXiv:hep-lat/0110168].

[4] M. Albanese et al. [APE Collaboration], Phys. Lett. B192, 163 (1987); M. Falcioni, M. L. Paciello, G. Parisi and B. Taglienti, Nucl. Phys. B251 (1985) 624.

[5] G. P. Lepage and P. B. Mackenzie, Phys. Rev. D 48, 2250 (1993) [arXiv:hep-lat/9209022].

[6] Some early references include T. DeGrand [MILC Collaboration], Phys. Rev. D58, 094503 (1998) [hep-lat/9802012]; Phys. Rev. D60, 094501 (1999) [hep-lat/9903006]; T. DeGrand, A. Hasenfratz and T. G. Kovacs, Nucl. Phys. B 547, 259 (1999) [hep-lat/9810061]. Fat links also have an extensive use in staggered fermion simulations; see J. F. Lagae and D. K. Sinclair, Phys. Rev. D 59, 014511 (1999) [hep-lat/9806014]; K. Orginos, D. Toussaint and R. L. Sugar [MILC Collaboration], Phys. Rev. D 60, 054503 (1999) [hep-lat/9903032]; and F. Knechtli and A. Hasenfratz, Phys. Rev. D 63, 114502 (2001) [arXiv:hep-lat/0012022].

[7] M. Ishibashi, Y. Kikukawa, T. Noguchi and A. Yamada, Nucl. Phys. B 576, 501 (2000) [arXiv:hep-lat/9911037].

[8] C. Alexandrou, E. Follana, H. Panagopoulos and E. Vicari, Nucl. Phys. B 580, 394 (2000) [arXiv:hep-lat/0002010].

[9] S. Capitani and L. Giusti, Phys. Rev. D 62, 114506 (2000) [arXiv:hep-lat/0007011].

[10] S. Capitani and L. Giusti, Phys. Rev. D 64, 014506 (2001) [arXiv:hep-lat/0011070].

[11] S. Capitani, Nucl. Phys. Proc. Suppl. 106, 826 (2002) [arXiv:hep-lat/0108028].

[12] C. W. Bernard and T. DeGrand, Nucl. Phys. Proc. Suppl. 83, 845 (2000) [arXiv:hep-lat/9909083].

[13] W. j. Lee and S. R. Sharpe, arXiv:hep-lat/0208018.

[14] W. j. Lee, arXiv:hep-lat/0208032.

[15] R. Gupta, T. Bhattacharya and S. R. Sharpe, Phys. Rev. D 55, 4036 (1997) [arXiv:hep-lat/9611023].

[16] K. Hornbostel, G. P. Lepage and C. Morningstar, Nucl. Phys. Proc. Suppl. 94, 579 (2001) [arXiv:hep-lat/0011049]; arXiv:hep-ph/0208224.

[17] C. Bernard et al. [MILC Collaboration], arXiv:hep-lat/0206016; a paper in preparation, by C. Bernard and T. Degrand. more fully describes the prescription.

[18] A. Patel and S. R. Sharpe, Nucl. Phys. B 395, 701 (1993) [arXiv:hep-lat/9210039].

[19] T. DeGrand, Ref. [6].

[20] G. P. Lepage, CLNS-80/447.

[21] G. Martinelli, Phys. Lett. B 141, 395 (1984).

[22] N. Tracas and N. Vlachos, Phys. Lett. B 115, 419 (1982).

[23] A. J. Buras, arXiv:hep-ph/9806471.

[24] C. W. Bernard, A. Soni and T. Draper, Phys. Rev. D 36, 3224 (1987).

[25] P. Weisz, private communication.

[26] W. Bietenholz, arXiv:hep-lat/0204016.

[27] P. Hasenfratz, S. Hauswirth, K. Holland, T. Jorg, F. Niedermayer and U. Wenger, Int. J. Mod. Phys. C 12, 691 (2001) [arXiv:hep-lat/0003013]; C. Gattringer, M. Gockeler, C. B. Lang, P. E. Rakow and A. Schafer, Phys. Lett. B 522, 194 (2001) [arXiv:hep-lat/0108001]. S. Hauswirth, Bern thesis, arXiv:hep-lat/0204015. P. Hasenfratz, S. Hauswirth, T. Jorg, F. Niedermayer and K. Holland, arXiv:hep-lat/0205010.

[28] T. DeGrand, work in progress. For preliminary results, see arXiv:hep-lat/0208054. 